\title{
Assessing the external exposome of South African children using wearable passive samplers and high- resolution mass spectrometry
}

Jeremy P. Koelmel

Yale University

Elizabeth Z. Lin

Yale University

Kayley DeLay

Yale University

Antony J. Williams

EPA: Environmental Protection Agency

Yakun Zhou

Yale University

Riana Bornman

University of Pretoria

Muvhulawa Obida

University of Pretoria

Jonathan Chevrier

McGill University

Krystal Pollitt ( $\nabla$ krystal.pollitt@yale.edu )

Yale University

\section{Research Article}

Keywords: Exposome, wristbands, children's health, Africa, exposure assessment, chemicals

Posted Date: August 23rd, 2021

DOl: https://doi.org/10.21203/rs.3.rs-828351/v1

License: (c) (1) This work is licensed under a Creative Commons Attribution 4.0 International License.

Read Full License 


\section{Abstract}

Children in low- and middle-income countries are often exposed to higher levels of, and more vulnerable to, the health effects of air pollution. Little is known about the diversity, toxicity, and dynamics of airborne chemical exposures at the molecular level.

We developed a workflow employing state-of-the-art wearable passive sampling technology coupled with high-resolution mass spectrometry to comprehensively measure 147 children's personal exposures to airborne chemicals in Limpopo, South Africa, as part of the VHEMBE study.

637 environmental exposures were detected, many of which have never been measured in this population, including 50 airborne chemical exposures of concern in the children, including biocides, plasticizers, organophosphates, dyes, combustion products, and perfumes. Biocides detected in wristbands included $p, p^{\prime}$-DDT, $p, p^{\prime}$-DDD, $p, p^{\prime}$-DDE, propoxur, piperonyl butoxide, and triclosan. $27 \%$ of exposures were significantly different across seasons. Our study provides the first report covering hundreds of chemical exposures among African children, demonstrating chemical exposures warranting further study.

\section{Introduction}

In 2016 it was estimated that $23 \%$ of the global disease burden was attributable to environmental factors (Prüss-Üstün and Corvalán 2007; Prüss-Üstün et al. 2016). Enhanced vulnerability was identified in low- and middle-income countries (LMIC) due to increasing chemical production and use, lack of regulation/enforcement and education on chemical exposure risks, and differences in priorities due to the need for economic development (Trasande et al. 2011a). Further concerns have been raised regarding the unique challenges in sub-Saharan Africa related to the lack of infrastructure for waste management and water treatment, poor availability of low-cost fuels, and high risk for malaria (Fayiga et al. 2018). Over $90 \%$ of rural populations in Africa burn refuse or biomass for cooking or heating. This practice can generate household air pollution (HAP), with fine particulate matter $\left(\mathrm{PM}_{2.5}\right)$ levels commonly exceeding that of the World Health Organization guidelines (Petkova et al. 2013; Vanker et al. 2015, 2017). Given the systemic inflammation and oxidative stress induced by $\mathrm{PM}_{2.5}$, elevated HAP exposure is ranked as the second environmental factor of concern in global burden of disease (Landrigan et al. 2018; Bigna and Noubiap 2019). In malaria endemic areas, the adverse effects of $\mathrm{PM}_{2.5}$ may be compounded by insecticides applied on the interior walls of homes through Indoor Residual Spraying (IRS) for malaria control. This practice can result in elevated exposure to dichlorodiphenyltrichloroethane (DDT) and pyrethroids which may interfere with immune function by inducing oxidative stress or by disrupting sex hormones (Pérez-Maldonado et al. 2005; Nasuti et al. 2007; Han et al. 2008; Meeker et al. 2009; Yoshinaga et al. 2014; Molina et al. 2021). Environmental exposure to insecticides from IRS in the Limpopo province of South Africa has recently been associated with accelerated fetal growth (Chevrier et al. 2019a), reduced social-emotional scores at 1 and 2 years of age (Eskenazi Brenda et al.), allergies in children at $3.5 \mathrm{yrs}$ (Huq et al. 2020), hypertensive disorders among pregnant mothers (Murray et al. 2018), 
weight gain in girls (Coker et al. 2018), and other health impacts (Bornman et al. 2016; Murray et al. 2018; Huang et al. 2018; Verner et al. 2018; Chevrier et al. 2019a, b; Huq et al. 2020; Eskenazi Brenda et al.; Huang Jonathan et al.).

Children from these rural regions of Africa often spend extended periods of time indoors, near cooking fires, which can result in high exposure to airborne pollutants. Exposure to environmental contaminants during critical windows of susceptibility coupled with poverty and malnutrition may enhance vulnerability (Suk et al. 2003; Sly and Flack 2008). Understanding the extent to which these factors contribute to poor health, requires a comprehensive assessment of environmental exposures. The studies that have been conducted to date in sub-Saharan Africa have focused on a limited panel of pesticides or regulated air pollutants (Bornman et al. 2016; Murray et al. 2018; Huang et al. 2018; Verner et al. 2018; Chevrier et al. 2019a, b; Huq et al. 2020; Eskenazi Brenda et al.; Huang Jonathan et al.). The lack of available technology which can be feasibly deployed with children in a LMIC context has presented challenges in the breadth and scale of environmental measurements that can be captured. We recently developed a lightweight, low-cost, and non-invasive device (the FreshAir wristband) to facilitate population-scale exposure assessment in LMICs (Figure 1A) (O'Connell et al. 2014; Hammel et al. 2016, 2018; Wang et al. 2019; Koelmel et al. 2020b, a; Lin et al. 2020). The FreshAir wristband is an exposomic sensor technology for evaluating an individual's personal exposure to contaminants from combustion, consumer products, clothing, and dietary (foods and additives) sources (Doherty et al. 2021). While the wristband does not directly measure the types of products and diet, mixtures of airborne chemicals measured can be used to specify what is being cooked, used, and worn. The device passively absorbs airborne chemicals into a polydimethylsiloxane (PDMS) sorbent membrane that is contained in a unique sheltered design that both minimizes effects of wind on uptake rates(Seethapathy et al. 2008; Zhang et al. 2013) and eliminates direct dermal contact (Figure 1B). This contained design is especially important for children who are prone to play with the passive samplers.

Coupling the wristband technology with gas chromatography high-resolution mass spectrometry (GCHRMS) enables evaluation of the occurrence, distribution, and magnitude of expected environmental contaminants of concern and screening for thousands of unexpected chemical exposures (Koelmel et al. 2020a). After chemical characterization of exposure profiles, newly introduced methods can be deployed to rapidly categorize detected compounds based on toxicity using experimental and computational evidence (Martin et al. 2008; BENFENATI et al. 2009; Zhu et al. 2009; Cassano et al. 2010; Williams et al. 2017; Vegosen and Martin 2020).

The objective of this study was to evaluate the environmental exposures of children in the Limpopo province of South Africa and identify the exposures of most concern. Exposures were evaluated for children between the ages of 5 and 6.5 years enrolled in the Venda Health Examination of Mothers, Babies and their Environment (VHEMBE) Birth Cohort using the Fresh Air wristband. While the health impacts of criteria air pollutants and insecticide use are recognized (Bornman et al. 2016; Murray et al. 2018; Huang et al. 2018; Verner et al. 2018; Chevrier et al. 2019a, b; Huq et al. 2020; Eskenazi Brenda et al.; Huang Jonathan et al.), other sources of environmental exposures may be missed in rural African 
populations. We determined likely chemical structures for 637 chemicals, including 50 chemical exposures of concern consisting of combustion products, insecticides and other biocides, dyes, fragrances, organophosphates, and plasticizers. This work demonstrates the feasibility of using novel exposomic sensors in a LMIC context, enabling a holistic exposure assessment approach to identify regionally specific environmental exposure signatures.

\section{Methods}

\subsection{Population, site characteristics, and study design}

This study leveraged ongoing research from the VHEMBE program, which began as a birth cohort in 2012 with 752 mother-child pairs from rural villages in the Vhembe district of Limpopo, South Africa. The overall aim of the VHEMBE program is to identify environmental determinants of health and sources of environmental contaminants to develop interventions to mitigate exposures. Pregnant women were recruited when they presented to give birth at Tshilidzini Hospital in the town of Thohoyandou between 2012 and 2013. Enrollment was restricted to women above the age of 18 years who spoke Tshivenda as their primary language at home, resided less than $20 \mathrm{~km}$ from the hospital and did not have intentions of moving away from the area within the following two years, were not infected with malaria during pregnancy, had contractions at least 5 minutes apart and gave birth to a live singleton. Further information about the VHEMBE program has been published previously. ${ }^{1}$

Chemical exposure was assessed for 147 children ages 5 to 6.5 years participating in the VHEMBE program between October 2018 to January 2019 and July 2019 to September 2019. Some families moved away from the immediate area (>20 km from Tshilidzini Hospital), requiring travel distances longer than 30 minutes to the homes. Exposures were evaluated using FreshAir wristbands worn over a 72-hour sampling period. Children were instructed to only remove samplers while bathing or swimming. At the end of the 72 hours (about 3 days), wristbands were collected.

The primary caregivers of study children completed structured questionnaires at the time of the child's birth as well as at 1 week and 1, 2, 3.5, and 5 years of age. Questionnaires were also administered to caregivers in Tshivenda at the time the FreshAir wristband were picked up, to collect data on participants' household characteristics, the date(s) and time(s) of cooking/burning materials, which fuels were used, whether fires occurred indoors or outdoors and whether the child was present during biomass burning, and household characteristics, including poverty status. Other information was recorded on the questionnaire such as the road and traffic conditions near their home, their proximity to large factories/warehouses or other major sources of pollution, the primary area of cooking, if a secondary cooking area was used, and GPS coordinates for cooking locations and the child's bedroom. All participants provided informed consent prior to data collection. This study was approved by Institutional Review Boards from McGill University, the University of Pretoria, the Limpopo Department of Health and Social Development, Tshilidzini Hospital and Yale University. 
2.2. Wristband preparation, deployment, and gas chromatography high resolution mass spectrometry (GC-HRMS) data-acquisition

Wristband preparation and data-acquisition have been previously described (Lin et al. 2020; Koelmel et al. 2020a). Briefly, PDMS sorbent bars were custom fabricated and cleaned in a vacuum oven $\left(2 \mathrm{~h}, 300^{\circ} \mathrm{C}\right)$ prior to use. PDMS sorbent bars were transported in airtight $2 \mathrm{~mL}$ amber glass vials with PTFE septa caps from Yale University to the study field office at Tshilidzini Hospital at room temperature. Immediately prior to deployment, study field staff inserted four pre-cleaned PDMS sorbent bars into the custom designed PTFE chambers and mounted these chambers into wristband attachments.

Participants were asked to remove the wristband while bathing or swimming. At the end of the exposure assessment period, study field staff collected and stored the FreshAir wristband in the air-tight container while being transported back to the study field office. Immediately after arrival, the field staff removed the PDMS sorbent bars from each sampler and placed them back into the airtight glass storage vials using stainless steel forceps. Samples were stored in a refrigerator at the study field office and transported using cold chain shipment to the Yale School of Public Health. Samples were then stored at $-20^{\circ} \mathrm{C}$ prior to analysis. Field blanks collected during the study were used to correct for potential contamination. These PDMS bars (field blanks) were processed similarly to participant samples, including both storage and placement in and out of PTFE chambers and wristband attachments (to simulate sample preparation), but were not placed on participants.

Though FreshAir wristbands were designed to capture gas-phase chemicals, particle deposition was observed on all PDMS sorbent bar samples (Figure 1). This observation was expected because only the main road in the study region was paved, leading to an increased amount of resuspended dust being captured by the FreshAir sampler during the participant's daily routine. Furthermore, biomass burning likely resulted in particle deposition. To remove excess dust debris, PDMS sorbent bars were rinsed immediately prior to analysis using Optima LC/MS grade water. PDMS sorbent bars were then spiked with an internal standard mixture which contained 4,4'-dibromooctafluorobiphenyl, 5'-fluoro-2,3',4,4',5pentabromodiphenyl ether, naphthalene-d8, 1-methylnaphthalene-d10, acenaphthene-d10, fluorene-d10, phenanthrene-d10, fluoranthene-d10, pyrene-d10, perylene-d12, phenol-d5, and p-terphenyl-d10. Sorbent bars were then placed into pre-cleaned glass autosampler tubes (Gerstel, Linthicum, MD, USA) on a temperature-controlled autosampler tray maintained at $10^{\circ} \mathrm{C}$ (MéCour, Groveland, MA, USA). For sample analysis, an autosampler tube was transferred into a thermal desorption unit (TDU; Gerstel, Linthicum, MD, USA). The TDU was initially held at $30^{\circ} \mathrm{C}$ for $1.1 \mathrm{~min}$ and then ramped at $720^{\circ} \mathrm{C}$ per minute to $280^{\circ} \mathrm{C}$ ( 5 min hold) under a flow rate of $350 \mathrm{~mL} / \mathrm{min}$ of helium gas (99.999\%). Extracted analytes were cyrofocused to $-90^{\circ} \mathrm{C}$ on a $2 \mathrm{~mm}$, glass wool deactivated liner in a cooled injection system (Gerstel, Linthicum, MD, USA) cooled to $-90^{\circ} \mathrm{C}$. The transfer line between the TDU and cooled liner was maintained at $250^{\circ} \mathrm{C}$. Analytes were directly transferred to the GC column (TG-5SILMS, 30m x $0.25 \mathrm{~mm} \times 0.25 \mu \mathrm{m}$; Thermo Fisher Scientific, Waltham, MA). The carrier gas flow (helium) was set to $1.4 \mathrm{~mL} / \mathrm{min}$ and the GC oven was held at $70{ }^{\circ} \mathrm{C}$ for one minute and then ramped at $7{ }^{\circ} \mathrm{C} / \mathrm{min}$ to $300^{\circ} \mathrm{C}$. The final temperature was held for 4.0 min for a total run-time of 37.86 minutes. During the analysis, full-scan electron ionization (EI) 
mass spectra $(\mathrm{m} / \mathrm{z} 53.4-800)$ were recorded at an acquisition rate of $4 \mathrm{~Hz}$, and at 60,000 resolution on a Q-Exactive Orbitrap mass spectrometer (ThermoFisher, Waltham, MA, USA). QCs and blanks (laboratory and transport) which were run every 5 samples.

\subsection{Suspect screening and data-processing workflow}

Data-processing consisted of spectral deconvolution, compound annotation, and alignment in Compound Discoverer 3.2 (Thermo Fisher Scientific, Waltham, MA). Compound Discoverer was used for the determination of the relative abundances and possible identifications (annotations) of compounds across all participants. In-house scripts performed filtering to determine the highest confidence annotations, remove noise and measured chemicals unrelated to exposures, and normalize the signal for comparison across instrument batches and between compounds. Alkanes were used to calculate Kovat's retention indices (Kováts 1958) for all features. The workflow has been described in-depth previously (Koelmel et al. 2020a).

The raw mass spectrometry datasets were first converted to a table of aligned features (retention times and associated deconvoluted fragments) using Compound Discoverer 3.2. The table consisted of chemical abundances across all samples and field blanks, tentative annotations using a composite score of retention index (RI) matching, a dot-product search index (SI) and reverse search index (RSI), highresolution mass filter (HRMF) and reverse high resolution mass filter (RHRMF). Tentative annotations were assigned using the NIST 2017, Wiley $11^{\text {th }}$ edition, and GC-Orbitrap (Thermo Fisher Scientific) EI spectral libraries, together covering over 740,000 unique chemical structures. All tentative annotations containing a calculated RI and library RI were removed if RI differences were higher than 100.

After processing in Compound Discoverer, the dataset was exported as an Excel file, and an in-house script was used to designate annotations as "high confidence", "medium confidence," or "low confidence." High confidence annotations had molecular ions observed if in the library, retention index matches within 50 units, RHRMF scores greater than 75, SI scores greater than 500, and RSI scores greater than 600 . These confident annotations accurately define the exact chemical structure or a closely resembling isomer (for example the methyl positions for dimethyl naphthalene will not be known). Medium confidence scores were those meeting the same criteria, except retention index and molecular ion matching. All the remaining scores were designated as low confidence. Manual review of 25 high confidence annotations of interest was performed to validate the method, as well as confirmation of the top hit from the scoring method using over 70 standards. Following ranking of confidence, blank feature filtering (BFF) (Patterson et al. 2017), duplicate removal (by name and CAS-RN), batch-wise median normalization, and TIC recalculation was performed as described previously (Koelmel et al. 2020a).

\subsection{Statistics and data interpretation}

Resulting annotations were ranked to determine potential chemicals of most concern. Predicted acute toxicity and AMES mutagenicity for screening likely carcinogens was performed using the US-EPA CompTox Chemicals Dashboard (Williams et al. 2017) (from here on referred to as the Dashboard) batch 
search function (using CASRN as inputs). Chemicals were ranked by predicted mammalian acute toxicity (rat oral LD 50,24 hours) (Zhu et al. 2009), developmental toxicity (Cassano et al. 2010) and mutagenicity (BENFENATI et al. 2009; Sushko et al. 2010) to determine the top compounds of concern. Furthermore, chemicals were screened to determine those chemicals deemed as high priority due to human health and environmental concerns by various governmental organizations using lists contained in the Dashboard (Williams et al. 2017). These lists included:

1. the EPA Toxics Release Inventory (Lynn and Kartez 1994; US EPA 2013), which lists 677 chemicals which either have acute or chronic human health effects (e.g., cancer causing compounds) or significant adverse environmental effects <https://comptox.epa.gov/dashboard/chemical_lists/TRIRELEASE>.

2. The Agency for Toxic Substances and Disease Registry (ATSDR) (U.S. Department of Health and Human Services) list contained in the Toxic Substances Portal (200 chemicals deemed toxic to human health) <https://comptox.epa.gov/dashboard/chemical_lists/ATSDRLST> (2020)

3. The Minnesota Department of Health Chemicals of High Concern and Priority Chemicals list which was established under the Toxic Free Kids act (1643 chemicals of health concern, especially to vulnerable population including children and pregnant mothers) <https://comptox.epa.gov/dashboard/chemical_lists/MNDOHTOXFREE> (Minnesota Department of Health 2019a)

4. The 20 high priority chemicals selected from the Toxic Substances Control Act Chemical (TSCA) Substance Inventory (41,587 active chemicals) (US EPA 2015) which are undergoing further review based on health concerns <https://comptox.epa.gov/dashboard/chemical_lists/TSCAHIGHPRI>.

5. The NORMAN European Food Safety Authority Priority Substances (178 chemicals of 2336 chemicals assessed which were selected based on toxicity, environmental release, lack of biodegradation, and bioaccumulation in food) <https://comptox.epa.gov/dashboard/chemical_lists/EFSAPRl> (Oltmanns et al. 2019).

6. The NORMAN Potential Persistent, Mobile and Toxic (PMT) substances (269 chemicals) <https://comptox.epa.gov/dashboard/chemical_lists/UBAPMT> (Örtl 2019).

Furthermore, chemicals with the highest inhalation and dermal toxicity, ocular irritation, and carcinogenicity were identified using the prototype EPA Hazard Comparison Dashboard (EPA-HCD), an application resulting from adding additional data and functionality to the reported Alternatives Assessment Dashboard (Vegosen and Martin 2020). This dashboard compiles both experimental and predicted toxicities from various organizations and categorized chemicals from low to very high in categories spanning both health hazards and chemical properties.

Multivariable analysis (Principal Component Analysis) and univariate analysis (ANOVA and volcano plots) were performed in Metaboanalyst 5 (Chong et al. 2018) to compare exposures across seasons. Participants were grouped based on date of sampling, with 99 participants falling under the dry season (July/August, with 8 of these 99 participants falling in the transition month of September). The remaining 
participants were classified as falling within the wetter season (October/November/December). Furthermore, levels of chemicals were compared across poverty and cooking fuel types and frequencies. For all univariate tests $p$-values were adjusted using the Hochberg method to correct for multiple testing errors. Food poverty (yes or no) was defined as having a household income below R547 per person per month when children were 5 years old based on Statistics South Africa guidelines.

\section{Results And Discussion}

Personal exposure assessment using the FreshAir wristband (Figure 1) is a relatively low-cost method for investigating the external exposome of vulnerable individuals including children. This study had a relatively high participation rate, attributable to the long-term partnerships between the study field staff and participants' families, as well as the comfortable and aesthetically pleasing design of the FreshAir wristbands. Early on in this study the PDMS sorbent bars of a few wristbands were lost because participants opened the wristband chamber. To minimize loss, and increase participation rate, we prevented the chamber from being opened using torx headed screws. The combination of this 'childproofed' design and a t-shirt offered as a reward for returning an intact wristband, increased sample recovery.

Future work is still needed to improve sample recovery and quality. Our study staff encountered numerous challenges trying to recover the wristbands. These included claims like the wristbands were lost and that other children had removed them; but our study children also tended to hide the bands from the staff. There was evidence of tampering with the device such as chew marks trying to open the cassettes and others left stones inside the cassettes. Screws were not always holding tight, or the mothers had to sew back the bands after they were torn. We also heard explanations such as that the children had lost the bands in the pit toilets after visiting the toilet. These challenges were probably reflecting the poor socio-economic conditions of the mothers and therefore, pre-emptive action should be taken in similar future studies.

Exposure profiles were assessed for 147 children enrolled in the VHEMBE study between the age of 5 and 6.5 years. Due to various sources of emissions of concerns, including cookstoves using plastic and biomass for cooking (Figure 1A and Figure 1B), unfiltered vehicle exhaust (Figure 1C), open refuse disposal and biomass and refuse burning (Figure 1D), and indoor and outdoor pesticide application (Figure 1E), a diverse array of exposures were expected. Thousands of chemical signatures from various sources were detected in these children, showing the breadth of coverage of this technique. Across the children that were included in the study, 3,580 chemicals were annotated after removing any signatures from transport or other background sources. Of these, 637 were assigned a chemical structure with "high confidence", meaning that the exact structure or a close structural isomer are likely the correct annotation (see methods). A further 1,209 chemical annotations were assigned with "medium confidence", meaning that the chemical class was likely correct, but the exact structure may not be (see the Supplemental Data for all detected compounds, confidence assignments, and relative abundances). 
Chemical exposures detected using the FreshAir wristbands were screened against chemical priority lists developed by various non-profit and government agencies in Europe and the United States to determine chemical exposures which may pose health risks (Figure 2). These lists consist of chemicals with acute or chronic toxic effects, or those suspectced of being toxic which were under further review by government agencies. Certain lists also accounted for environmental fate and transport and the exposure potential to each chemical. Of special relevance was the Minnesota Department of Health Chemicals of High Concern and Priority Chemicals List which was established under the Toxic Free Kids (TFK) Act and consists of 1,643 chemicals (referred to here on out as the "TFK list") (Minnesota Department of Health 2019a). The TFK List contains chemicals of health concern, especially to vulnerable populations such as children. Forty-six of the 637 chemical exposures assigned with high confidence were found in at least one of the six priority lists, with 20 occurring in 2 or more lists, and 37 occurring on the TFK list (Figure 2). These chemicals were found across multiple sources including biocides (e.g., insecticides), plasticizers (e.g., phthalates), combustion products (polycyclic aromatic hydrocarbons (PAHs) and furans), flame retardants, fragrances, dyes, and personal care products (Figure 2). This shows the diverse sources of chemicals of potential health concern in this rural population of South Africa.

The lists of chemicals of concern screened in this study were developed by government agencies and organizations in the United States and Europe. Interestingly, these children in remote African villages are exposed to a plethora of synthetic chemicals also impacting children in developed countries, including flame retardants, (e.g., tris(2-carboxyethyl) phosphine (TCEP), tris(2-chloroisopropyl) phosphate (TCPP), and triphenyl phosphate) (Figure 2). The priority lists developed in high income countries may not reflect the chemical risks of those living in developing countries, because exposure potential in the two settings may be different. Furthermore, developing and emerging economies use, and production of manmade chemicals is increasing overtime, and local communities often do not have the funding to characterize these chemical exposures in a non-targeted fashion.(Trasande et al. 2011b) Therefore, as in this study, personal exposures must be characterized across various cultural, economic, and geographically diverse populations in order to better understand potential chemicals of concern from a high exposure standpoint. Furthermore, priority lists should account for these diverse populations, so that region specific lists of chemicals of concern can be more effectively deployed, without missing chemicals which do not appear in Western countries with different regulations, for example. Finally, experimental toxicity values can then account for a wide range of chemicals which are not prioritized in the West.

Chemicals were additionally screened by predicted oral acute toxicity and mutagenicity, as well as compiled experimental evidence of "very high" acute inhalation toxicity, dermal toxicity, carcinogenicity, and ocular irritation using the EPA-HCD (Vegosen and Martin 2020) (Figure 3). The hazard scores of low $(L)$, moderate $(M)$, high $(H)$, or very high $(V H)$ hazard represented in the EPA-HCD are based on the DfE Alternatives Assessment Criteria for Hazard Evaluation which provides guidance for converting values from a variety of different sources and formats into the consistent $L, M, H$, and VH scores (US EPA Design for the environment program alternatives assessment criteria for hazard evaluation Version 2.0. https://www.epa.gov/sites/production/files/2014-01/documents/aa_criteria_v2.pdf.). "Very high" is the highest category of toxicity on the EPA-HCD. Carcinogenic compounds that the children were exposed to 
that were categorized as "very high" toxicity included those from combustion products (PAHs: chrysene, benzo(k)fluoranthene, and benz(a)anthracene), phthalates, and components of insecticides used to control malaria in the villages including $p, p^{\prime}$-DDT and its degradation product, $p, p^{\prime}-\mathrm{DDE}$ (dichlorodiphenyl dichloroethylene) (Figure 3). Chemicals with exceedingly high acute dermal toxicity detected included benzothiazole (used in car tires and found in other consumer/industrial products) and piperonyl butoxide (used as a synergist to enhance insecticide efficacy against malaria carrying mosquitoes in Africa (Gleave et al. 2018a)). Inhalation toxicity is of special relevance in this study. The FreshAir wristband design has a perforated chamber that eliminates direct contact of the PDMS sorbent bar with the skin; any chemicals monitored were volatile or semi-volatile chemicals in the air. Therefore, all chemicals detected are likely being inhaled by the children to some degree. Of great concern are the five chemicals detected with very high acute inhalation toxicity (pyrene, p-cresol, triclosan, and propoxur; Figure 3).

Insecticides are sprayed on the interior walls of residences in the study region to control malaria through IRS practices; DDT is primarily applied to interior walls of unpainted mud dwellings and the pyrethroids deltamethrin or cypermethrin are primarily applied to homes with painted walls. (Bouwman et al. 2006) DDT and its breakdown product, DDE has been detected in serum collected from the mothers of VHEMBE participants (Gaspar Fraser W. et al.). $p, p^{\prime}$-DDT and $p, p^{\prime}$-DDE were also detected by FreshAir wristbands worn by children in the current study. $p, p^{\prime}$-DDD, DEET, piperonyl butoxide (Gleave et al. 2018b) and propoxur (Gericke A. et al. 2002) were additionally observed, which are all active ingredients in insecticides, insecticide transformation products, or used in mosquito repellant products. Most children had detectable levels of these insecticides and insecticide synergists (Figure 3), with, for example, all insecticides, insecticide synergists, and insecticide transformation products were detected in $75 \%$ or more of the participants (Figure 3 and Figure 4 C). The distributions of these chemicals were similar across participants (Figure 3), with most children having pesticide exposure within 2 orders of magnitude of each other (Figure 3). To our knowledge, while over 40 pesticide exposures have been measured in Africa (Anderson et al. 2014; Jepson et al. 2014; Donald et al.), including airborne exposures to $p, p^{\prime}$-DDT and $p, p^{\prime}-\mathrm{DDE}$, this is the first study to measure exposures to propoxur, piperonyl butoxide, and DEET, as well as triclosan, another biocide, in rural Africa. These unique surrogate measurements of inhaled chemical exposures in children raise concern, as both propoxur and triclosan likely have very high acute inhalation toxicity as determined by the EPA-HCD.

Another source of chemical exposure for these children was from combustion of wood and refuse used for cooking in huts with little ventilation (Figure 2). Overall, over 10 plasticizers (including phthalates) and over 150 potential PAHs were detected (See Supplemental Data), showing the vast diversity of exposures children have to chemicals falling under these classes of compounds. A substantial portion of chemicals were significantly higher in abundance for children whose parents reported longer durations of cooking time ( $>8$ hours) compared to those with no cooking or shorter cooking times ( $<4$ hours) during the sampling period. Plasticizer exposures, on the other hand, were not correlated to cooking time suggesting combustion may not be the major source. It is interesting to note, that when comparing poverty across individuals for each seasonal period, participants with higher poverty had higher exposure to certain combustion products (e.g., benzo[k]fluoranthene) suggesting poverty may play a role in exposure to these 
toxic chemicals (Figure S1). Whereas total PAHs and individual PAHs have been monitored in Africa, exposures to most PAHs determined in this study have not been previously reported in exposure studies in Africa (Chimuka et al. 2016). Hence further work looking into the complex array of individual PAHs and their potential health impacts on these children is warranted, as PAH toxicity can drastically differ depending on chemical species (Gao et al. 2015; Geier et al. 2018).

Exposures of concern among the children differed from child to child, often ranging several orders of magnitude (Figure 3). Some of the chemicals with the highest variance in exposure levels across children were chemicals with specific uses to which children may not be universally exposed: these included active ingredients of pesticides, nicotyrine (a metabolite of nicotine), and caffeine. Caffeine had the widest range in exposure levels across children, ranging across 7 orders of magnitude. Comparing between chemicals, the phthalates had the highest signal of all detected compounds. For example, the average signal across all participants for all 637 chemicals was highest for di(2-ethylhexyl) phthalate, followed by butyl isobutyl phthalate ranked as $7^{\text {th }}$ and diisobutyl phthalate as $8^{\text {th }}$ highest exposure compound.

Exposures also differed across seasons for the cohort studied. Limpopo, South Africa, has two main seasons: the wet, hot season and the dry, cold season. PCA showed distinct personal exposure profiles as a factor of season, suggesting that seasonality was the main force influencing variation in measured exposure profiles (Figure 4A). Of the 637 chemicals confidently annotated, 27\% (171) varied significantly across season (Hochberg corrected p-value $<0.05$ and a greater than 2-fold change difference) (Figure 4B). The vast majority of these 171 chemicals were higher in the dry season (78\%).

During the dry season, dust may be dispersed by wind, vehicles, and by other means, increasing the load of particulate matter captured by the passive samplers. Furthermore, the lower level of numerous chemicals during the wet season, including certain chemicals of concern, is attributed to wet deposition of airborne pollutants by precipitation (Jayamurugan et al. 2013; Olszowski 2016). An example is shown for exposure levels of common volatile wood and plant emissions (longipinene, D-limonene, and 3carene) with $\mathrm{D}$-limonene and 3-carene decreasing by at least an order of magnitude on average during the wet season (Figure 4C). In contrast, certain chemicals of concern were elevated during the wet season suggesting environmental changes and/or seasonal changes in behaviors enhancing their emission or exposure. Active ingredients in insecticides (e.g., propoxur and $p, p^{\prime}$-DDT) and mosquito repellant (e.g., DEET) were all significantly increased during the warmer wet season when mosquito populations are higher, suggesting increased insecticide use in the wet season (Figure 4C). Field observations also show that this is the season when indoor residual spraying is conducted.

While this study focused on chemicals of potential health hazard for the children in the villages studied, the chemical profiles measured could be used to answer several questions related to the children's behavior and environment. For example, numerous flavor related compounds were measured, as well as active ingredients in beverages (e.g., caffeine) and a metabolite in cigarettes (nicotyrine) which could help assess the children's or family's diet/activities. Certain chemicals detected (e.g., nylon-6) could determine 
the type of plastics used or combusted by the community or family (depending on proximity to other villagers). Detected dye related chemicals (e.g., indigo dye, quinaldines and quinolines) and fragrances (e.g., musk ketone and galaxolide) could provide information on social factors and behaviors. Compounds such as dimethyl indole which was detected could be related to exposure to fecal matter (major odor of fecal matter).

Indeed, certain compounds which may have specific emission sources were found to correlate with poverty in either the wet or dry season. For example, lilial, a compound used in perfumes and laundry detergent, was detected at higher levels in participants during classified as less poor during the dry season. During the wet season lilial abundances approached significance for those less poor versus remaining children (Figure S1). Camphor exposure was also higher in the dry season for participants classified as above the poverty threshold (Figure S1). Chloroxylenol, an antiseptic used in soaps and other consumer products to kill bacteria, was higher in participants in the wet season classified as below the poverty threshold (Figure S1). Several combustion products (e.g., dimethyl-naphthalene, acenaphthylene, 2-ethenyl-napthalene, benzo(k)fluoranthene, and pyrroles) were higher in participants classified as below the poverty threshold in one or both seasons (Figure S1). This is likely due to the higher use of solid fuel for cooking used by this group and lower level of ventilation.

\section{Conclusion}

We show that the FreshAir wristbands, a low-cost technology, can be used to passively capture airborne contaminants (Lin et al. 2020), enabling measurement of a portion of the chemical exposome of vulnerable populations in LMICs. More research is needed to assess exposure profiles of children in developing countries. This work highlights the range of chemicals of concern to which children in rural regions of South Africa are exposed. Bridging this technology together with state-of-the-art gas chromatography high-resolution mass spectrometry enables the screening of hundreds of thousands of chemicals to determine both expected and unexpected exposures in different populations. Personal exposures to 637 chemicals assigned with relatively high confidence were measured in the VHEMBE birth cohort, many of which have not previously been measured as airborne exposures in Africa at large. Fifty were deemed of potential health concern based on priority lists developed by governments based on human health hazards, predicted toxicity values, and experimentally derived toxicity values. These chemicals of concern include components of insecticides used for malaria control (e.g., $p, p^{\prime}$-DDT, $p, p^{\prime}$ $\mathrm{DDD}, p, p^{\prime}$-DDE and piperonyl butoxide), PAHs from combustion (with over 150 individual PAHs detected), plasticizers including phthalates, organophosphate compounds, fragrances, dyes, and biocides (e.g., triclosan). Because the wristband is designed to primarily detect airborne chemicals (gases and particles), it is of additional concern that several chemical exposures measured are classified as having very high acute inhalation toxicity. These chemicals included pyrene, p-toly(4-methylbenzyl) sulfide, pcresol, triclosan, and propoxur. Furthermore, many chemicals do not have data pertaining to inhalation toxicity, or the data is inconclusive, showing the need for efforts to assign inhalation toxicity values for these airborne chemicals. 
Individual exposure profiles for chemicals of concern ranged across orders of magnitude from individual to individual and were significantly dependent on season. Twenty-seven percent of the confidently annotated chemicals significantly differed across season, with most chemicals decreasing in abundance during the wet-hot season. Certain chemicals, such as $p, p^{\prime}$-DDT, DEET, and propoxur used for mosquito control increased in the wet-hot season, which is aligned with the increased use of insecticides and mosquito repellant during the wetter warmer season to deal with malaria. Furthermore, certain chemical exposures were significantly different depending on degree of poverty and cooking methods, showing the ability to assess the effect of socio-economic status and behaviors on exposure profiles using this technology.

This study assessed the wide breadth of chemical exposures of children in rural regions of South African including numerous chemicals which have not previously been measured as airborne exposures in this region; however, this likely represents only the tip of the iceberg. Other complimentary analysis such as liquid chromatography high-resolution mass spectrometry could further extend coverage of assessed exposures. Follow up quantitative analysis and analysis of chemicals in the urine and blood are planned to assess whether the levels of these chemicals are of substantial concern. Furthermore, linking exposure data to specific participant activities, consumer product use, housing characteristics, and environmental factors will be helpful to determine potential interventions for reducing exposure.

\section{Declarations}

Funding: This work was supported by the Canadian Institute for Health Research and a Canada Research Chair in Global Environmental Health and Epidemiology (to JC).

Ethics approval: This study was approved by Institutional Review Boards from McGill University, the University of Pretoria, the Limpopo Department of Health and Social Development, Tshilidzini Hospital and Yale University.

Consent to participate: Mothers and/or primary caregivers provided informed consent prior to data collection.

Conflicts of interest/Competing interests: All authors declare no competing interests or conflicts of interest.

Consent for publication: All authors provide consent for publication according to the policy set out by Exposure and Health.

Data Availability: Raw mass spectrometry data is available upon request. Processed data is available as an excel as part of the supplemental.

Disclaimer: The views expressed in this paper are those of the authors and do not necessarily reflect the views or policies of the U.S. Environmental Protection Agency or the Canadian Institute of Health 
Research. Mention of trade names or commercial products does not constitute endorsement or recommendation for use.

\section{References}

Anderson KA, Seck D, Hobbie KA, et al (2014) Passive sampling devices enable capacity building and characterization of bioavailable pesticide along the Niger, Senegal and Bani Rivers of Africa. Philos Trans R Soc Lond B Biol Sci 369:20130110. https://doi.org/10.1098/rstb.2013.0110

BENFENATI E, BENIGNI R, DEMARINI DM, et al (2009) Predictive Models for Carcinogenicity and Mutagenicity: Frameworks, State-of-the-Art, and Perspectives. Journal of Environmental Science and Health, Part C 27:57-90. https://doi.org/10.1080/10590500902885593

Bigna JJ, Noubiap JJ (2019) The rising burden of non-communicable diseases in sub-Saharan Africa. The Lancet Global Health 7:e1295-e1296. https://doi.org/10.1016/S2214-109X(19)30370-5

Bornman MS, Chevrier J, Rauch S, et al (2016) Dichlorodiphenyltrichloroethane exposure and anogenital distance in the Venda Health Examination of Mothers, Babies and their Environment (VHEMBE) birth cohort study, South Africa. Andrology 4:608-615. https://doi.org/10.1111/andr.12235

Bouwman H, Sereda B, Meinhardt HM (2006) Simultaneous presence of DDT and pyrethroid residues in human breast milk from a malaria endemic area in South Africa. Environmental Pollution 144:902-917. https://doi.org/10.1016/j.envpol.2006.02.002

Cassano A, Manganaro A, Martin T, et al (2010) CAESAR models for developmental toxicity. Chem Cent J 4:S4. https://doi.org/10.1186/1752-153X-4-S1-S4

Chevrier J, Rauch S, Crause M, et al (2019a) Associations of Maternal Exposure to Dichlorodiphenyltrichloroethane and Pyrethroids With Birth Outcomes Among Participants in the Venda Health Examination of Mothers, Babies and Their Environment Residing in an Area Sprayed for Malaria Control. American Journal of Epidemiology 188:130-140. https://doi.org/10.1093/aje/kwy143

Chevrier J, Rauch S, Obida M, et al (2019b) Sex and poverty modify associations between maternal peripartum concentrations of DDT/E and pyrethroid metabolites and thyroid hormone levels in neonates participating in the VHEMBE study, South Africa. Environment International 131:104958. https://doi.org/10.1016/j.envint.2019.104958

Chimuka L, Sibiya P, Amdany R, et al (2016) Status of PAHs in Environmental Compartments of South Africa: A Country Report. Polycyclic Aromatic Compounds 36:376-394.

https://doi.org/10.1080/10406638.2014.988276

Chong J, Soufan O, Li C, et al (2018) MetaboAnalyst 4.0: towards more transparent and integrative metabolomics analysis. Nucleic Acids Research 46:W486-W494. https://doi.org/10.1093/nar/gky310 
Coker E, Chevrier J, Rauch S, et al (2018) Association between prenatal exposure to multiple insecticides and child body weight and body composition in the VHEMBE South African birth cohort. Environment International 113:122-132. https://doi.org/10.1016/j.envint.2018.01.016

Doherty BT, Koelmel JP, Lin EZ, et al (2021) Use of Exposomic Methods Incorporating Sensors in Environmental Epidemiology. Curr Envir Health Rpt 8:34-41. https://doi.org/10.1007/s40572-021-003068

Donald CE, Scott RP, Blaustein KL, et al Silicone wristbands detect individuals' pesticide exposures in West Africa. Royal Society Open Science 3:160433. https://doi.org/10.1098/rsos.160433

Eskenazi Brenda, An Sookee, Rauch Stephen A., et al Prenatal Exposure to DDT and Pyrethroids for Malaria Control and Child Neurodevelopment: The VHEMBE Cohort, South Africa. Environmental Health Perspectives 126:047004. https://doi.org/10.1289/EHP2129

Fayiga AO, Ipinmoroti MO, Chirenje T (2018) Environmental pollution in Africa. Environ Dev Sustain 20:41-73. https://doi.org/10.1007/s10668-016-9894-4

Gao B, Wang X-M, Zhao X-Y, et al (2015) Source apportionment of atmospheric PAHs and their toxicity using PMF: Impact of gas/particle partitioning. Atmospheric Environment 103:114-120. https://doi.org/10.1016/j.atmosenv.2014.12.006

Gaspar Fraser W., Chevrier Jonathan, Quirós-Alcalá Lesliam, et al Levels and Determinants of DDT and DDE Exposure in the VHEMBE Cohort. Environmental Health Perspectives 125:077006. https://doi.org/10.1289/EHP353

Geier MC, Chlebowski AC, Truong L, et al (2018) Comparative developmental toxicity of a comprehensive suite of polycyclic aromatic hydrocarbons. Arch Toxicol 92:571-586. https://doi.org/10.1007/s00204017-2068-9

Gericke A., Govere J.M., Durrheim D.N. (2002) Insecticide susceptibility in the South African malaria mosquito Anopheles arabiensis (Diptera: Culicidae) : research letter. South African Journal of Science 98:205-208. https://doi.org/10.10520/EJC97445

Gleave K, Lissenden N, Richardson M, et al (2018a) Piperonyl butoxide (PBO) combined with pyrethroids in insecticide-treated nets to prevent malaria in Africa. Cochrane Database Syst Rev 11:CD012776. https://doi.org/10.1002/14651858.CD012776.pub2

Gleave K, Lissenden N, Richardson M, et al (2018b) Piperonyl butoxide (PBO) combined with pyrethroids in insecticide-treated nets to prevent malaria in Africa. Cochrane Database Syst Rev 11:CD012776. https://doi.org/10.1002/14651858.CD012776.pub2

Hammel SC, Hoffman K, Webster TF, et al (2016) Measuring Personal Exposure to Organophosphate Flame Retardants Using Silicone Wristbands and Hand Wipes. Environ Sci Technol 50:4483-4491. 
Hammel SC, Phillips AL, Hoffman K, Stapleton HM (2018) Evaluating the Use of Silicone Wristbands To Measure Personal Exposure to Brominated Flame Retardants. Environ Sci Technol 52:11875-11885. https://doi.org/10.1021/acs.est.8b03755

Han Y, Xia Y, Han J, et al (2008) The relationship of 3-PBA pyrethroids metabolite and male reproductive hormones among non-occupational exposure males. Chemosphere 72:785-790.

https://doi.org/10.1016/j.chemosphere.2008.03.058

Huang Jonathan, Eskenazi Brenda, Bornman Riana, et al Maternal Peripartum Serum DDT/E and Urinary Pyrethroid Metabolite Concentrations and Child Infections at 2 Years in the VHEMBE Birth Cohort. Environmental Health Perspectives 126:067006. https://doi.org/10.1289/EHP2657

Huang JY, Eskenazi B, Bornman R, et al (2018) Maternal peripartum urinary pyrethroid metabolites are associated with thinner children at 3.5 years in the VHEMBE birth cohort (Limpopo, South Africa). Environ Epidemiol 2:e026. https://doi.org/10.1097/EE9.0000000000000026

Huq F, Obida M, Bornman R, et al (2020) Associations between prenatal exposure to DDT and DDE and allergy symptoms and diagnoses in the Venda Health Examination of Mothers, Babies and their Environment (VHEMBE), South Africa. Environmental Research 185:109366. https://doi.org/10.1016/j.envres.2020.109366

Jayamurugan R, Kumaravel B, Palanivelraja S, Chockalingam MP (2013) Influence of Temperature, Relative Humidity and Seasonal Variability on Ambient Air Quality in a Coastal Urban Area. International Journal of Atmospheric Sciences 2013:e264046. https://doi.org/10.1155/2013/264046

Jepson PC, Guzy M, Blaustein K, et al (2014) Measuring pesticide ecological and health risks in West African agriculture to establish an enabling environment for sustainable intensification. Philosophical Transactions of the Royal Society B: Biological Sciences 369:20130491. https://doi.org/10.1098/rstb.2013.0491

Koelmel JP, Lin EZ, Guo P, et al (2020a) Exploring the external exposome using wearable passive samplers - the China BAPE study. Environmental Pollution 116228.

https://doi.org/10.1016/j.envpol.2020.116228

Koelmel JP, Lin EZ, Nichols A, et al (2020b) Head, shoulders, knees, and toes: Placement of wearable passive samplers alters exposure profiles observed. Environmental Science and Technology Submitted:

Kováts E (1958) Gas-chromatographische Charakterisierung organischer Verbindungen. Teil 1: Retentionsindices aliphatischer Halogenide, Alkohole, Aldehyde und Ketone. Helvetica Chimica Acta 41:1915-1932. https://doi.org/10.1002/hlca.19580410703 
Landrigan PJ, Fuller R, Acosta NJR, et al (2018) The Lancet Commission on pollution and health. The Lancet 391:462-512. https://doi.org/10.1016/S0140-6736(17)32345-0

Lin EZ, Esenther S, Mascelloni M, et al (2020) The Fresh Air Wristband: A Wearable Air Pollutant Sampler. Environ Sci Technol Lett. https://doi.org/10.1021/acs.estlett.9b00800

Lynn FM, Kartez JD (1994) Environmental democracy in action: The Toxics Release Inventory. Environmental Management 18:511-521. https://doi.org/10.1007/BF02400856

Martin TM, Harten P, Venkatapathy R, et al (2008) A hierarchical clustering methodology for the estimation of toxicity. Toxicol Mech Methods 18:251-266. https://doi.org/10.1080/15376510701857353

Meeker JD, Barr DB, Hauser R (2009) Pyrethroid insecticide metabolites are associated with serum hormone levels in adult men. Reprod Toxicol 27:155-160.

https://doi.org/10.1016/j.reprotox.2008.12.012

Minnesota Department of Health (2019a) Toxic Free Kids Act: Chemicals of High Concern - EH: Minnesota Department of Health

Molina EM, Kavazis AN, Mendonça MT (2021) Chronic Exposure to Environmental DDT/DDE in 2 Species of Small Rodents: Measures of Contaminant Load, Immune Dysfunction, and Oxidative Stress. Environmental Toxicology and Chemistry 40:1619-1629. https://doi.org/10.1002/etc.5007

Murray J, Eskenazi B, Bornman R, et al (2018) Exposure to DDT and hypertensive disorders of pregnancy among South African women from an indoor residual spraying region: The VHEMBE study.

Environmental Research 162:49-54. https://doi.org/10.1016/j.envres.2017.12.006

Nasuti C, Gabbianelli R, Falcioni ML, et al (2007) Dopaminergic system modulation, behavioral changes, and oxidative stress after neonatal administration of pyrethroids. Toxicology 229:194-205. https://doi.org/10.1016/j.tox.2006.10.015

O'Connell SG, Kincl LD, Anderson KA (2014) Silicone Wristbands as Personal Passive Samplers. Environ Sci Technol 48:3327-3335. https://doi.org/10.1021/es405022f

Olszowski T (2016) Changes in PM10 concentration due to large-scale rainfall. Arab J Geosci 9:160. https://doi.org/10.1007/s12517-015-2163-2

Oltmanns J, Bohlen M-L, Escher S, et al (2019) Final Report: Applying a tested procedure for the identification of potential emerging chemical risks in the food chain to the substances registered under REACH - REACH 2. EFSA Supporting Publications 16:1597E. https://doi.org/10.2903/sp.efsa.2019.EN1597

Örtl E (2019) REACH: Improvement of guidance and methods for the identification and assessment of PMT/vPvM substances. Umweltbundesamt 
Patterson RE, Kirpich AS, Koelmel JP, et al (2017) Improved experimental data processing for UHPLCHRMS/MS lipidomics applied to nonalcoholic fatty liver disease. Metabolomics 13:142. https://doi.org/10.1007/s11306-017-1280-1

Pérez-Maldonado IN, Herrera C, Batres LE, et al (2005) DDT-induced oxidative damage in human blood mononuclear cells. Environmental Research 98:177-184. https://doi.org/10.1016/j.envres.2004.11.001

Petkova EP, Jack DW, Volavka-Close NH, Kinney PL (2013) Particulate matter pollution in African cities. Air Qual Atmos Health 6:603-614. https://doi.org/10.1007/s11869-013-0199-6

Prüss-Üstün A, Corvalán C (2007) How Much Disease Burden can be Prevented by Environmental Interventions? Epidemiology 18:167-178. https://doi.org/10.1097/01.ede.0000239647.26389.80

Prüss-Üstün A, Wolf J, Organization WH, et al (2016) Preventing Disease Through Healthy Environments: A Global Assessment of the Burden of Disease from Environmental Risks. World Health Organization

Seethapathy S, Górecki T, Li X (2008) Passive sampling in environmental analysis. Journal of Chromatography A 1184:234-253. https://doi.org/10.1016/j.chroma.2007.07.070

Sly PD, Flack F (2008) Susceptibility of Children to Environmental Pollutants. Annals of the New York Academy of Sciences 1140:163-183. https://doi.org/10.1196/annals.1454.017

Suk WA, Murray K, Avakian MD (2003) Environmental hazards to children's health in the modern world. Mutation Research/Reviews in Mutation Research 544:235-242.

https://doi.org/10.1016/j.mrrev.2003.06.007

Sushko I, Novotarskyi S, Körner R, et al (2010) Applicability domains for classification problems: Benchmarking of distance to models for Ames mutagenicity set. J Chem Inf Model 50:2094-2111. https://doi.org/10.1021/ci100253r

Trasande L, Massey RI, DiGangi J, et al (2011a) How developing nations can protect children from hazardous chemical exposures while sustaining economic growth. Health Aff (Millwood) 30:2400-2409. https://doi.org/10.1377/hlthaff.2010.1217

Trasande L, Massey RI, DiGangi J, et al (2011b) How developing nations can protect children from hazardous chemical exposures while sustaining economic growth. Health Aff (Millwood) 30:2400-2409. https://doi.org/10.1377/hlthaff.2010.1217

US EPA O (2013) Toxics Release Inventory (TRI) Program. In: US EPA. https://www.epa.gov/toxicsrelease-inventory-tri-program. Accessed 21 Dec 2020

US EPA O (2015) How to Access the TSCA Inventory. In: US EPA. https://www.epa.gov/tscainventory/how-access-tsca-inventory. Accessed 21 Dec 2020 
Vanker A, Barnett W, Nduru PM, et al (2015) Home environment and indoor air pollution exposure in an African birth cohort study. Science of The Total Environment 536:362-367.

https://doi.org/10.1016/j.scitotenv.2015.06.136

Vanker A, Barnett W, Workman L, et al (2017) Early-life exposure to indoor air pollution or tobacco smoke and lower respiratory tract illness and wheezing in African infants: a longitudinal birth cohort study. The Lancet Planetary Health 1:e328-e336. https://doi.org/10.1016/S2542-5196(17)30134-1

Vegosen L, Martin TM (2020) An automated framework for compiling and integrating chemical hazard data. Clean Techn Environ Policy 22:441-458. https://doi.org/10.1007/s10098-019-01795-w

Verner M-A, Chevrier J, Ngueta G, et al (2018) Early-life exposure to p,p'-DDT and p,p'-DDE in South African children participating in the VHEMBE study: An assessment using repeated serum measurements and pharmacokinetic modeling. Environment International 119:478-484.

https://doi.org/10.1016/j.envint.2018.07.010

Wang S, Romanak KA, Stubbings WA, et al (2019) Silicone wristbands integrate dermal and inhalation exposures to semi-volatile organic compounds (SVOCs). Environment International 132:105104. https://doi.org/10.1016/j.envint.2019.105104

Williams AJ, Grulke CM, Edwards J, et al (2017) The CompTox Chemistry Dashboard: a community data resource for environmental chemistry. J Cheminform 9:61. https://doi.org/10.1186/s13321-017-0247-6

Yoshinaga J, Imai K, Shiraishi H, et al (2014) Pyrethroid insecticide exposure and reproductive hormone levels in healthy Japanese male subjects. Andrology 2:416-420. https://doi.org/10.1111/j.20472927.2014.00202.x

Zhang X, Brown TN, Ansari A, et al (2013) Effect of Wind on the Chemical Uptake Kinetics of a Passive Air Sampler. Environ Sci Technol 47:7868-7875. https://doi.org/10.1021/es401486f

Zhu H, Martin TM, Ye L, et al (2009) QSAR Modeling of Rat Acute Toxicity by Oral Exposure. Chem Res Toxicol 22:1913-1921. https://doi.org/10.1021/tx900189p

(2020) Agency for Toxic Substances and Disease Registry. https://www.atsdr.cdc.gov/index.html. Accessed 21 Dec 2020

Toxic Free Kids Act: Chemicals of High Concern and Priority Chemicals - EH: Minnesota Department of Health. https://www.health.state.mn.us/communities/environment/childenvhealth/tfka/index.html. Accessed 21 Dec 2020b

Piperonyl Butoxide General Fact Sheet. http://npic.orst.edu/factsheets/pbogen.html. Accessed 19 Jun 2021c 


\section{Figures}

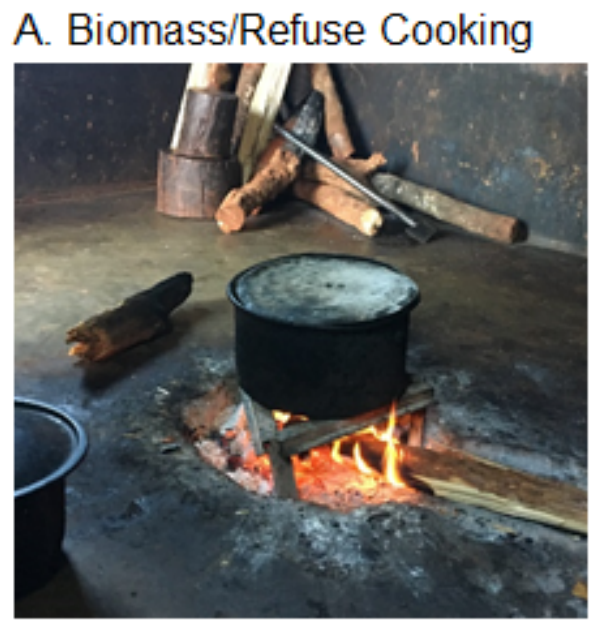

\section{B. Soot and Smoke Stains from Indoor Cooking}

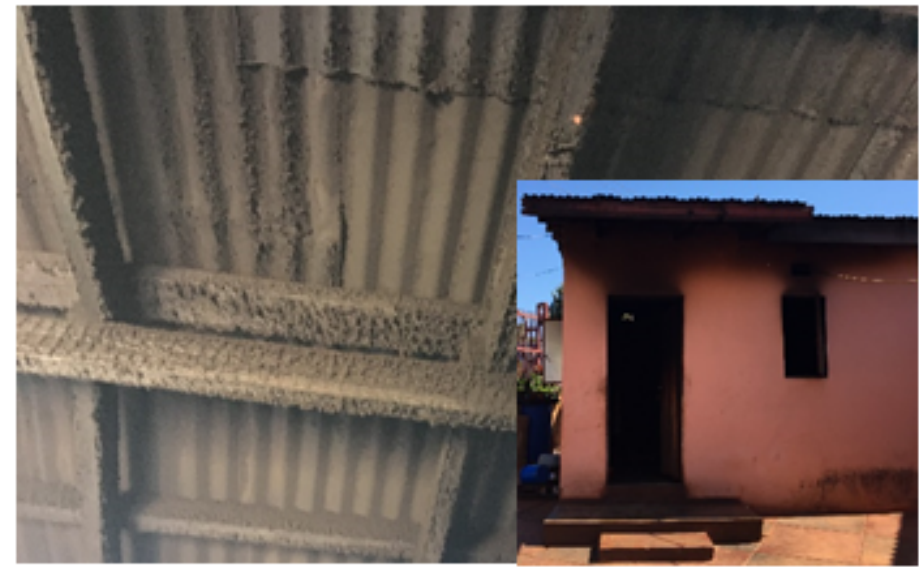

C. Vehicle Exhaust
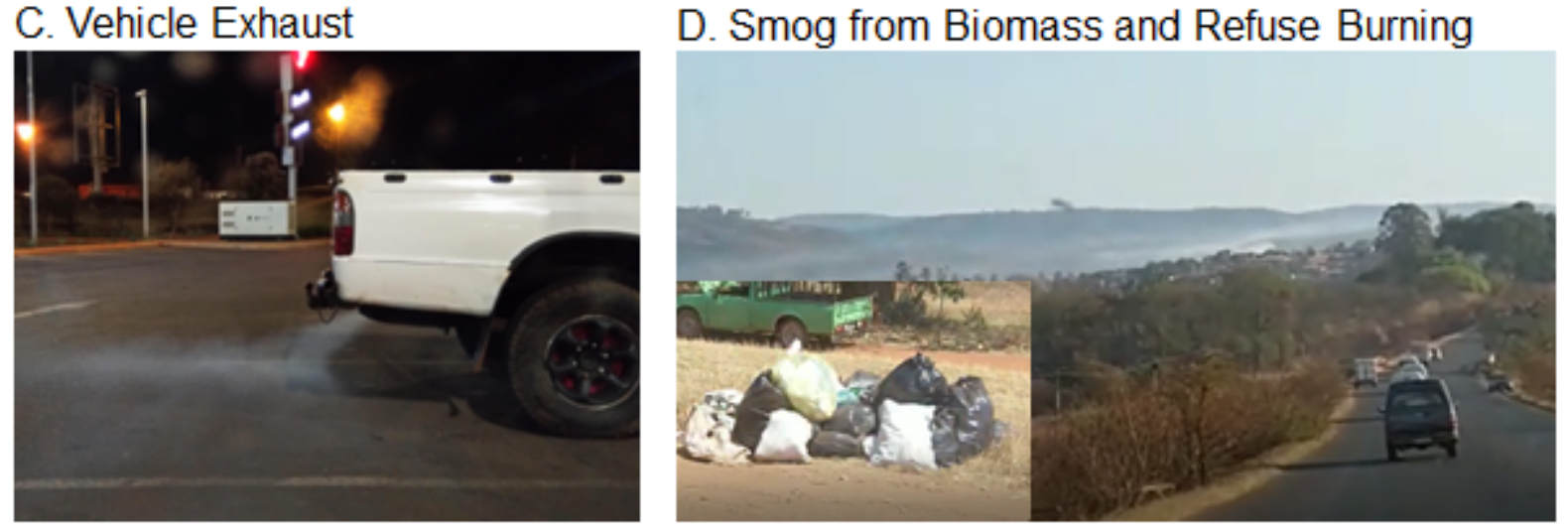

E. Indoor and Outdoor Insecticide Use

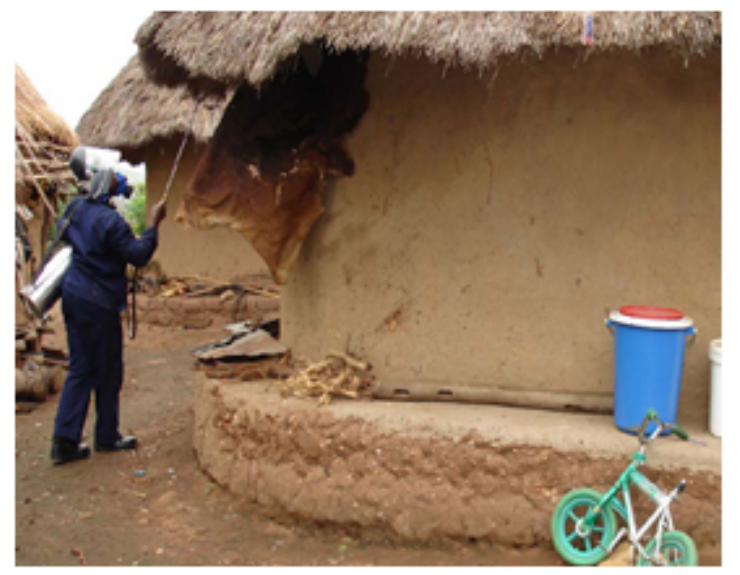

\section{F. Wristband Design and Application}

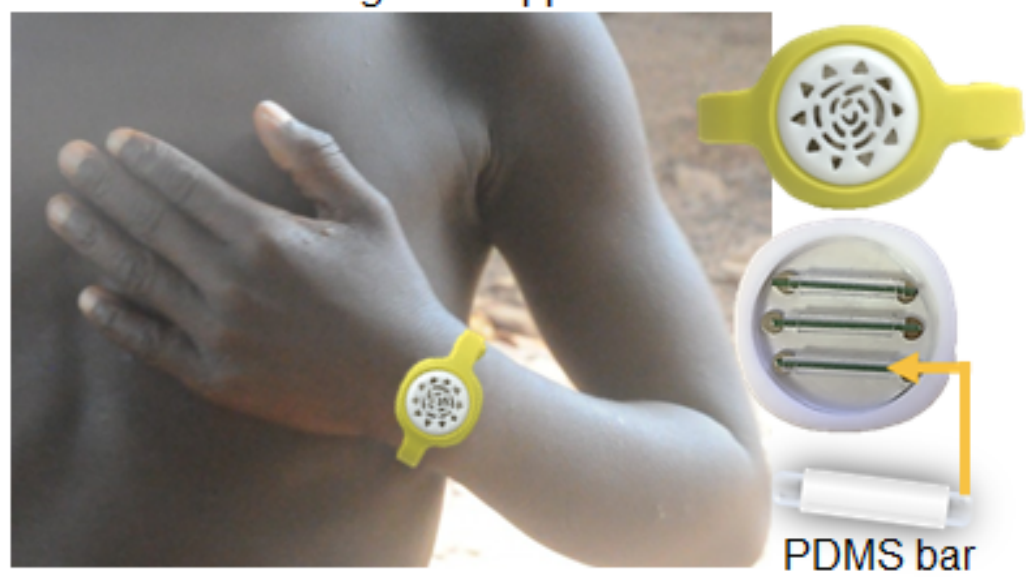

\section{Figure 1}

Images from the across the Limpopo region: an example of a cookstove that is typical of the region where biomass, plastic, and other refuse are burned for fuel (A), soot building up indoors on the roof and smoke stains from indoor cooking (B), vehicle tailpipe exhaust without emissions controls (C), smog from combustion of biomass (cooking) and plastics (refuse disposal) (D), residual spraying of pesticides which occurs indoors and outdoors $(E)$, as well as the FreshAir wristband $(F)$. The wristband consists of a 
PTFE chamber that contains custom fabricated PDMS sorbent bars, which passively absorb airborne contaminants.

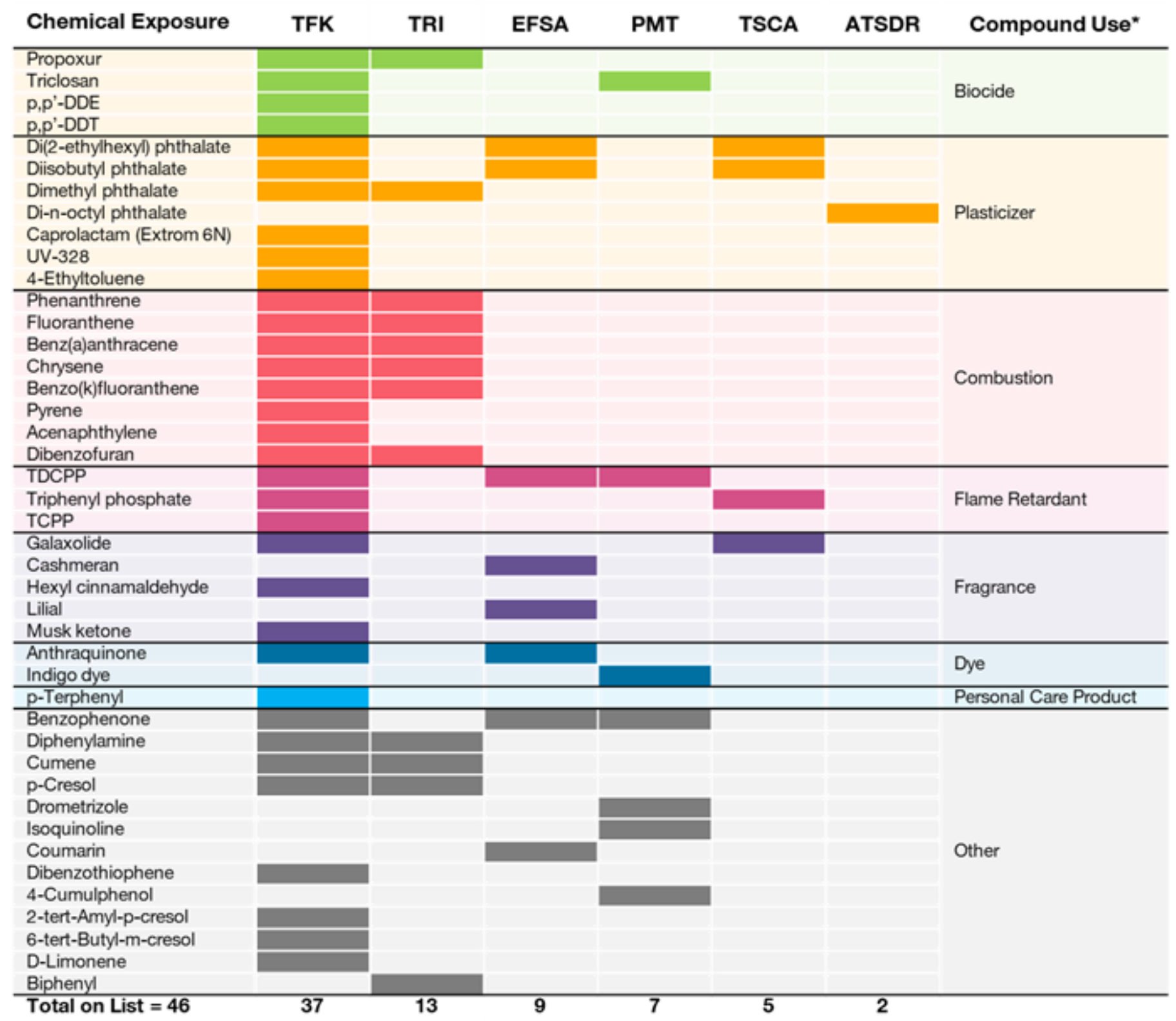

\section{Figure 2}

Forty-six chemical exposures detected in South African children which are listed in the following priority lists: NORMAN Potential Persistent, Mobile and Toxic (PMT) substances (269 chemicals), the NORMAN European Food Safety Authority (EFSA) Priority Substances (178 chemicals), the 20 high priority chemicals selected from the Toxic Substances Control Act Chemical (TSCA) Substance Inventory, EPA Toxics Release Inventory (TRI), which lists 677 chemicals which either have acute or chronic human health effects, The Agency for Toxic Substances and Disease Registry (ATSDR) which lists 200 chemicals deemed toxic to human health, and the Minnesota Department of Health Chemicals of High Concern and Priority Chemicals list which was established under the Toxic Free Kids (TFK) act (1643 chemicals of health concern, especially to vulnerable populations). Other acronyms: p,p'dichlorodiphenyltrichloroethane (p,p'-DDT), p,p'-dichlorodiphenyldichloroethylene (p,p'-DDE), tris(2- 
carboxyethyl)phosphine (TCEP), and tris(2-chloroisopropyl)phosphate (TCPP). *Note that common sources/uses of chemicals are provided, but these are not the only source of these chemicals and may not be the main sources of these chemicals in this population

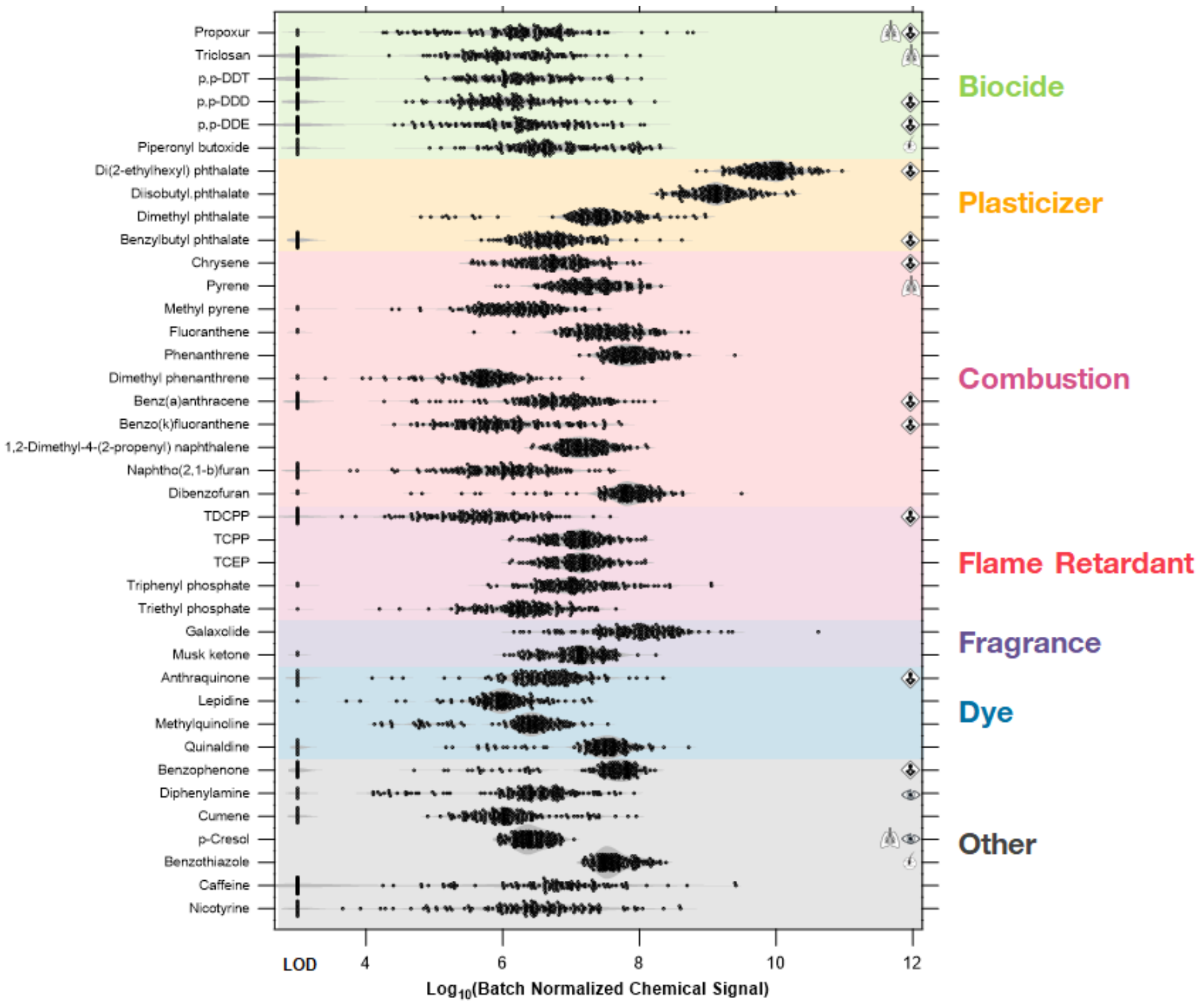

Health Hazards

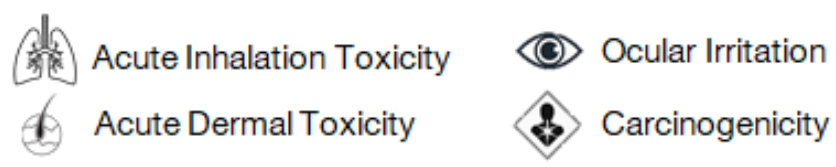

\section{Figure 3}

Thirty-five chemicals of potential concern. These chemical exposures of concern were highlighted based on occurrence in two or more priority lists (see methods) and/or the top 10 compounds with the highest predicted acute toxicity (inhalation, dermal)), ocular irritation, and carcinogenicity (predicted AMES mutagenicity) . Acronyms: Dichlorodiphenyltrichloroethane (p,p'-DDT), dichlorodiphenyldichloroethane (p,p'-DDD), dichlorodiphenyldichloroethylene (p,p'-DDE), tris(2-carboxyethyl)phosphine (TCEP), Tris(2- 
chloroisopropyl)phosphate (TCPP), and limit of detection (LOD). *Note that common sources/uses of chemicals are provided, but these are not the only source of these chemicals and may not be the main sources of these chemicals in this population

A. Volcano plot of exposures comparing the wet and dry season

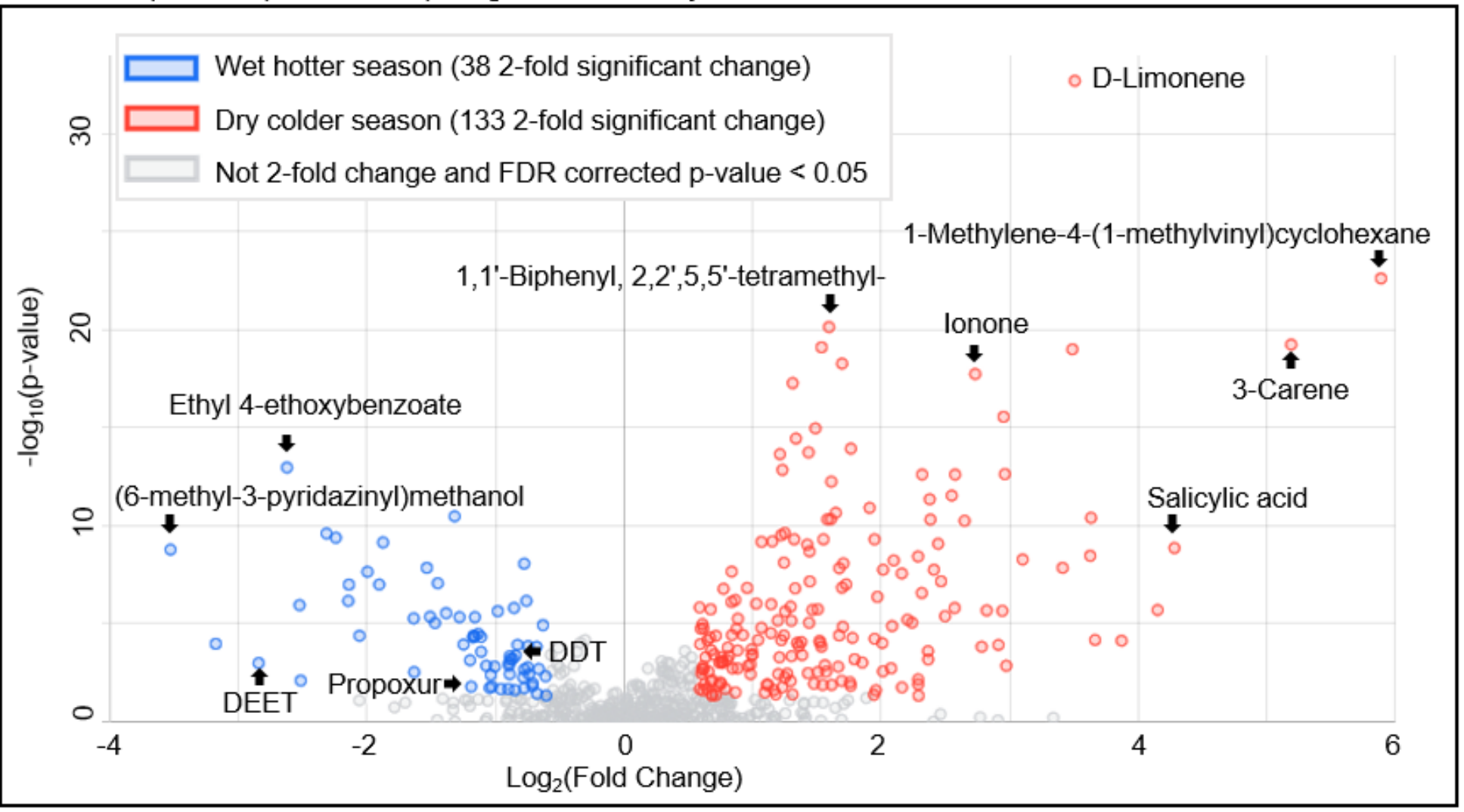

B. PCA scores of individuals colored by season

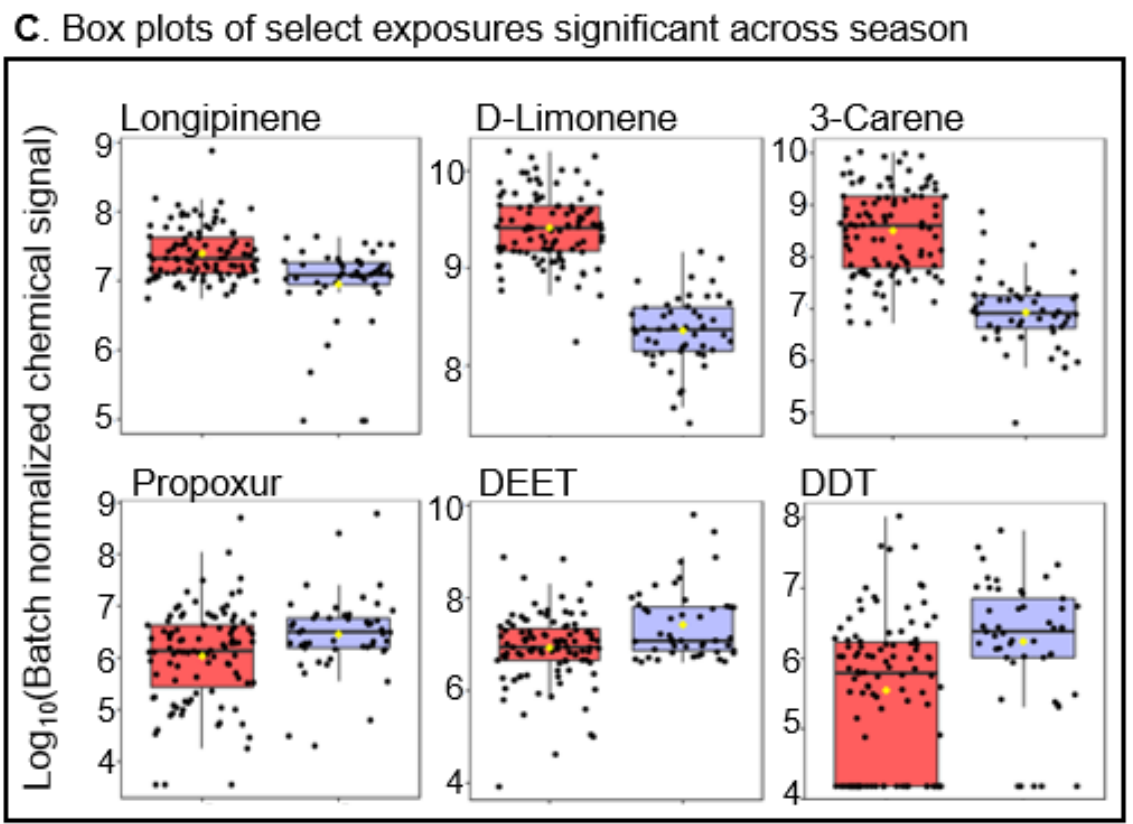

Figure 4

Comparison of chemical exposures in the wet, hot and dry, cold season. A) The volcano plot shows most exposures were increased during the dry season, and that a significant portion of chemical exposures were dramatically different depending on season. B) Principle components analysis (PCA) of exposures 
indicating distinct chemical profiles by season . C) Specific chemicals of interest are shown which were significant across season, specifically insecticide and insect repellant related chemicals, and wood emission related chemicals. Acronyms: Dichlorodiphenyltrichloroethane (p,p'-DDT), N, N-Diethyl-metatoluamide (DEET), and false-discovery rate (FDR).

\section{Supplementary Files}

This is a list of supplementary files associated with this preprint. Click to download.

- SUPPLEMENTALINFORMATION.docx

- 20210818AfricaCDSupplemental.xlsx 